La revue La revue pour l'histoire du CNRS

POUR L'HISTOIRE DU CNRS

1 | 1999

Le CNRS au temps de Charles de Gaulle

\title{
Le CNRS au temps de Charles de Gaulle
}

Le CNRS : principal enjeu de la politique scientifique

\section{Girolamo Ramunni}

\section{OpenEdition}

\section{Journals}

Édition électronique

URL : https://journals.openedition.org/histoire-cnrs/480

DOI : 10.4000/histoire-cnrs.480

ISSN : 1955-2408

Éditeur

CNRS Éditions

Édition imprimée

Date de publication : 6 novembre 1999

ISBN : 978-2-271-05707-5

ISSN : 1298-9800

Référence électronique

Girolamo Ramunni, "Le CNRS au temps de Charles de Gaulle ", La revue pour l'histoire du CNRS [En ligne], 1 | 1999, mis en ligne le 18 décembre 2006, consulté le 20 mai 2021. URL : http://

journals.openedition.org/histoire-cnrs/480 ; DOI : https://doi.org/10.4000/histoire-cnrs.480

Ce document a été généré automatiquement le 20 mai 2021

Comité pour l'histoire du CNRS 


\title{
Le CNRS au temps de Charles de Gaulle
}

\author{
Le CNRS : principal enjeu de la politique scientifique
}

\section{Girolamo Ramunni}

1 Un climat de confiance régnait parmi les scientifiques français au moment du retour aux affaires du général de Gaulle en 1958. Ils pensaient pouvoir dialoguer avec un homme qui, à la Libération, s'était inquiété du développement de la recherche en France. Ses prises de position par la suite ne laissaient planer aucun doute sur l'importance qu'il accordait à la recherche. Les scientifiques s'étaient donc mobilisés en vue d'approcher le Général ou les hommes de son entourage. Au CNRS, le nouveau directeur, Jean Coulomb, ne pouvait qu'être confiant. Depuis un an (12 juin 1957), il menait une évaluation discrète des laboratoires. Aussi la priorité accordée à la recherche laissait-elle espérer une expansion du Centre. Pour mener à bien la politique scientifique, le général de Gaulle avait cru nécessaire de créer de nouvelles structures, avant même d'accéder à la présidence de la République. Un Comité consultatif à la recherche scientifique et technique (CCRST), composé de douze personnalités, les Sages ${ }^{1}$, préparait et proposait au gouvernement des mesures en faveur de la recherche. Un délégué à la recherche scientifique et technique, rattaché au Premier ministre, était chargé de l'exécution des décisions prises par le gouvernement. Les grandes décisions étaient arrêtées lors de la tenue du Conseil interministériel de la recherche, auquel participaient les Sages et le délégué, outre les ministres concernés. Le premier gouvernement de la $\mathrm{V}^{\mathrm{e}}$ République comportait un ministre d'État chargé de la recherche, Louis Jacquinot, et un ministre de l'Éducation nationale, André Boulloche. Le CNRS était rattaché au ministre de l'Éducation nationale et pouvait être considéré comme l'un des organismes "spécialisés », comme l'Institut national de la recherche agronomique ou le Commissariat à l'énergie atomique. Dans la perspective d'une politique de la recherche coordonnée au niveau gouvernemental, le CNRS devait trouver sa place et prouver son originalité.

Depuis la Libération la recherche est une priorité 
2 Depuis la Libération, surtout à partir du milieu des années cinquante, les scientifiques s'étaient mobilisés pour que la recherche devienne une priorité nationale, ce qui souvent voulait dire non seulement «l'expansion illimitée » des crédits attribués à la recherche, mais surtout une expansion suivie sur plusieurs années. Les propositions de nouveaux organigrammes, la création de nouvelles institutions faisaient partie de ces temps forts qui avaient eu lieu au cours des débats lors du colloque de Caen. Parrainé par Mendès France, ce colloque (1-3 novembre 1956) avait été souhaité afin de discuter $\mathrm{du}$ "problème essentiel et urgent de la recherche scientifique en France » et pour «proposer des solutions »². Ensuite, s'était tenue, les 3, 4, 5 octobre 1957, à Grenoble, un colloque sur la politique de recherche industrielle. Ce fut au cours de cette réunion que les scientifiques français ont appris la nouvelle du lancement du Spoutnik. Cela fut immédiatement interprété comme une preuve supplémentaire du retard français. L'urgence devenait le maître mot. Que faire alors que la IV ${ }^{e}$ République était malmenée?

3 Cette même année, l'inspecteur des Finances Kientz déposait son rapport sur le financement des laboratoires de recherche dépendant des universités établi à la demande de R. Billères, ministre de l'Éducation nationale ${ }^{3}$. Au cours de sa mission, le rapporteur avait cru nécessaire d'analyser aussi les "établissements assimilés ». Le CNRS en faisait partie, parce qu'il mettait à la disposition des universitaires des chercheurs, du personnel technique contractuel et leur fournissait des subventions pour l'achat de matériels. C'était le lien étroit qui existait entre CNRS et Université. Kientz ne s'était pas limité à la gestion des crédits ; il avait estimé nécessaire d'étendre la comparaison au statut des personnels. De son enquête, il résultait que la situation des chercheurs était « unanimement considérée comme peu satisfaisante puisqu'ils n'ont ni l'avantage de la fonction publique (stabilité, retraite, possibilité de cumul), ni les rémunérations du secteur privé, sur lequel sont plus ou moins alignés les salaires d'établissements industriels et commerciaux comme le Commissariat à l'énergie atomique et l'office national d'études et de recherches aéronautiques...» Cette remarque est intéressante car elle montre la difficile situation du CNRS, considéré par certains comme une institution devant apporter un soutien naturel à l'Université, sans en avoir les bénéfices, et par d'autres comme un organisme de recherche devant entretenir des liens avec le monde de l'industrie, sans que les chercheurs aient les mêmes bénéfices que ceux des autres institutions de recherche ${ }^{4}$.

4 Faisant siennes les suggestions du colloque de Grenoble, Kientz demandait une modification des règles budgétaires «des établissements de haut enseignement universitaire et du CNRS » et la création d'organismes mixtes, université-industrie, de droit privé, dont les membres auraient dû être des industriels et des professeurs. Bref, le rapport entre recherche universitaire et industrie paraissait à ce moment l'élément essentiel du renouveau de la recherche; pour favoriser ce changement, les normes administratives des institutions d'enseignement supérieur devaient être changées. Déjà les participants au colloque de Grenoble avaient pris appui sur les "réalisations anglaises et américaines " pour demander un changement dans la réglementation en France. Kientz, reprenant l'idée de la possibilité d'avoir à l'Université des- .chercheurs sur contrats, concluait son rapport en précisant que, du point de vue administratif, il n'y avait pas d'obstacle à ce que les laboratoires universitaires puissent « recruter des agents contractuels ", d'autant plus qu'ils le faisaient déjà, comme le montrait le large usage que la faculté des sciences de Paris faisait « de cette possibilité dont le maintien 
paraît nécessaire... » Rédigé peu de temps avant le changement politique conduisant à la $V^{e}$ République, ce rapport mettait en lumière un point faible du CNRS : l'absence de statut pour les chercheurs. Il existait deux possibilités : calquer le statut des chercheurs CNRS sur celui des enseignants-chercheurs universitaires, ou sur celui des chercheurs d'organismes à vocation industrielle. On voit bien que la question du statut des personnels soulevait celle de la mission de l'organisme. En effet, nombreux étaient ceux qui pensaient que le CNRS n'avait pas pu accomplir jusque-là sa mission à cause de l'absence de statut, dont souffraient ses chercheurs. De ce point de vue, le rapport de Kientz ne faisait que reprendre certains thèmes déjà avancés à Caen.

Réorganiser la recherche en France

Il n'est pas étonnant de retrouver ces mêmes arguments parmi les premières propositions discutées au sein du CCRST. La réforme des structures de la recherche était une priorité et l'attention se portait avant tout sur le CNRS. Les opinions étaient fort diverses et chacun ne faisait au début, que reprendre ce qu'il avait déjà proposé, en d'autres occasions, au sujet du Centre. Un point semblait faire l'unanimité : il fallait radicalement modifier l'organisation de la recherche et commencer par réformer en profondeur le CNRS.

Charles Sadron fut l'un des premiers à proposer une solution. Il distinguait entre recherche fondamentale, activité devant être développée essentiellement sous l'égide de l'État, et recherche appliquée qui, pour se développer, avait besoin d'un cadre " moins bien défini ${ }^{5}$. Après une rapide analyse de l'état de la situation, il concluait que la recherche fondamentale était celle qui se faisait à l'Université et au CNRS. Considérant que les professeurs de l'enseignement supérieur ne pouvaient pas consacrer leur temps à la recherche comme cela aurait été souhaitable, car trop absorbés par les tâches d'enseignement, Sadron définissait le CNRS comme « une forme allotropique de l'enseignement supérieur, plus souple et mieux adaptée à la recherche scientifique fondamentale ». À la suite de ce bilan, il formulait le principe à partir duquel opérer le changement : il lui paraissait peu logique de garder inchangées deux structures si proches, ayant toutes les deux comme objectif de s'occuper de recherche fondamentale et fonctionnant en vase clos au sein du même ministère.

7 Sadron défendait une organisation de la recherche en centres puissants, en véritables " usines de recherches, destinées à entrer en concurrence avec les grands complexes scientifiques étrangers ", de 80 à 100 personnes, dont " une quinzaine ou une vingtaine de chercheurs qualifiés et permanents, donc de profession ». Les chercheurs du CNRS devaient pouvoir travailler dans ces centres, à la condition de leur octroyer un statut "assurant leur profession", car le fonctionnement de tels centres ne pouvait pas dépendre des fluctuations d'une carrière incertaine. Les centres pouvaient accueillir des thésards, mais ces jeunes devaient rester une minorité car la recherche performante ne pouvait pas s'appuyer sur une masse de jeunes apprentis. À côté de ces centres répondant à des objectifs et des priorités à caractère national, il fallait faire une place à la recherche que Sadron qualifiait d'indépendante. Cette recherche devait être organisée en instituts et en centres de recherche. Les premiers devaient être constitués par le regroupement de plusieurs laboratoires universitaires sous la direction d'un professeur, les seconds devaient rassembler plusieurs chercheurs travaillant dans une même discipline et constituer ce qu'on appelait autrefois une école. Le CNRS pouvait participer à ces centres et instituts en y envoyant des chercheurs non permanents. C'est là que se situait la distinction essentielle. Le CNRS était donc destiné à se détacher 
progressivement de la recherche universitaire pour venir renforcer la recherche appliquée, celle qui devait rendre des comptes à la Nation, qui n'avait pas la liberté de se diversifier selon le libre choix des chercheurs. La proposition de Sadron semble s'inspirer de l'organisation de la recherche allemande d'avant la guerre. Les « usines de recherches» appliquées rappellent les instituts du Kaiser Wilhelm Gesellschaft. Elle avait néanmoins un point faible car rien n'était dit à propos des autres organismes de recherche existant en France. De même, aucune indication n'était fournie quant aux structures envisagées pour coordonner et pour orienter l'action des instituts de recherche appliquée. Bref, la proposition était plutôt une esquisse d'organisation qu'un réel projet.

8 André Lichnérowicz, professeur de mathématiques au Collège de France, partait d'un diagnostic différent considérait que contrairement à ce qui se faisait partout ailleurs, la fonction de l'Université en France avait été, de tout temps, limitée à la double tâche de former des fonctionnaires de l'enseignement secondaire et de «cultiver l'esprit des honnêtes gens ${ }^{6} »$. Il proposait de rompre avec une tradition, remontant au XVIII ${ }^{e}$ siècle, qui faisait « résoudre tout problème d'enseignement spécialisé ou de recherche par la création d'organismes ad hoc en dehors des universités et à l'ombre d'un ministère utilisateur. Cette politique, qui était celle de la paresse d'esprit, s'était révélée profondément néfaste pour le pays: prolifération d'organismes agissant en mode dispersé se pensant comme de petites facultés autonomes, se combattant ou se coalisant pour neutraliser les universités ». Ce constat, formulé comme un réquisitoire contre une situation de facilité, ne devait pas faire oublier qu'en dépit de toutes les difficultés, les universités continuaient à faire de la recherche. Ce qui manquait était une coordination. Lichnérowicz demandait que soit créé un fonds national de la recherche fondamentale, destiné à soutenir la recherche universitaire, et des centres de recherche fondamentale spécialisée, confiés au CNRS. Ces centres auraient pu passer des contrats avec l'Université, Une condition était indispensable à la réussite de cette organisation: la titularisation des chercheurs du CNRS. Le mathématicien demandait aussi une expansion de l'Université avec la création d'universités scientifiques, issues de la transformation des anciennes facultés de sciences. Il n'est pas étonnant de trouver quelques éléments communs entre les deux projets, car Sadron et Lichnérowicz avaient participé au colloque de Caen (1956) et adhéré au Mouvement national pour le développement scientifique, créé en février 1958 par Marc Zamansky, professeur à la faculté des sciences de Paris.

9 Si l'on résume l'essentiel des deux projets, deux points leur sont communs : a) faire un tri et séparer les laboratoires qui travaillent en recherche fondamentale de ceux qui font de la recherche appliquée; b) doter les chercheurs du CNRS d'un statut. Ce deuxième point était considéré essentiel pour mener à bien la nouvelle politique. Voilà pourquoi Sadron avait cru nécessaire de protester au moment où le ministre de l'Éducation nationale s'apprêtait à prendre un décret créant un corps de maîtres assistants et donnant, à ceux qui s'y engageaient, la possibilité de faire une carrière complète. À son avis, cette mesure, prise de manière isolée, créait un obstacle supplémentaire à la reconnaissance d'une carrière pour les chercheurs $\mathrm{CNRS}^{7}$. Sadron demandait donc que l'on repousse cette réforme au moment où le statut dés chercheurs CNRS serait prêt. Le débat s'enlise 
Lors de la réunion du 21 janvier 1959, les Sages du CCRST n'étaient pas parvenus à s'entendre sur un projet d'organisation de la recherche. Un document avait été préparé, faisant le point des trois projets résumant l'essentiel des propositions formulées pendant les débats, Ce document leur fut soumis en février 1959, Pierre Piganiol avait proposé que les crédits consacrés à la recherche soient analysés dans leur globalité par le Comité interministériel de la recherche, et que leur gestion soit confiée aux divers ministères de tutelle des organismes de recherche. La création d'un fonds de la recherche, géré par le délégué, devait permettre des interventions urgentes mais limitées. Le projet de Piganiol reposait sur la nécessaire coordination entre les structures déjà en place. Il conçut l'enveloppe recherche, dont l'objectif était de présenter l'ensemble des crédits consacrés par l'État à la recherche en un document unique, indépendamment des ministères qui géraient effectivement ces crédits et les inscrivaient dans leur budget. Pour Piganiol, la priorité était d'arriver à mobiliser les chercheurs sur de nouvelles lignes de recherche, indépendamment de leurs organismes d'appartenance.

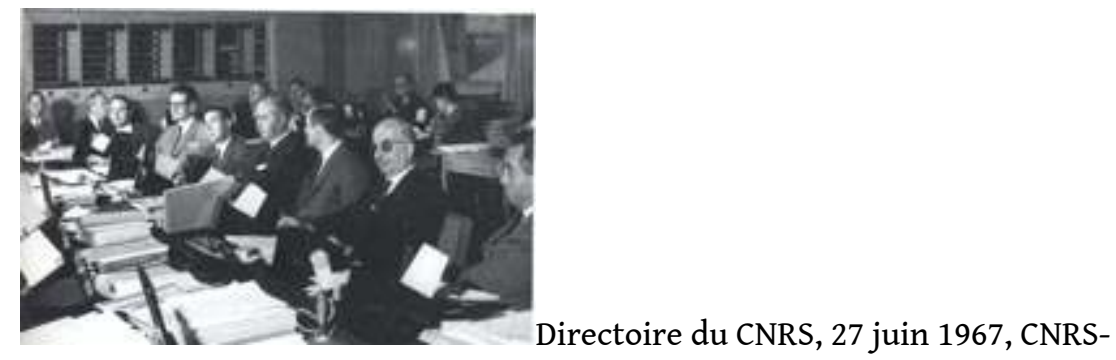

Photothèque.

11 La proposition de Sadron constituait le deuxième projet. Elle allait dans le sens opposé à celle de Piganiol : regroupement de tous les budgets et organismes de recherche sous une direction unique, rattachée de préférence au Premier ministre ; restructuration de l'ensemble des organismes de recherche à commencer par le CNRS. Une troisième solution avait été avancée par Paul Germain, professeur de mécanique, secrétaire perpétuel de l'Académie des sciences : laisser à chaque ministère la gestion des crédits, mais constituer un fonds de la recherche élargi, dont les crédits seraient répartis par un organisme interministériel dépendant du délégué et du CCRST, et pouvant utiliser le " comité national de la recherche scientifique (actuellement au CNRS) élargi et modifié dans sa composition, comme organisme consultatif ${ }^{8} »$. La solution proposée par Germain paraissait être un compromis entre celle de Sadron, très structurée en instituts, et celle, plus souple, de Piganiol.

Refusant de soutenir l'une des deux solutions extrêmes, le CCRST choisissait de travailler sur la proposition de Germain et commençait par déterminer les organismes destinés à prendre en charge les laboratoires. Les Sages penchaient pour la création d'un office fédérateur des gros centres de recherche ou office central de la recherche scientifique et technique (OCRST). regroupant « non seulement les gros laboratoires de recherche du CNRS mais aussi ceux des autres ministères ", et un office universitaire correspondant "plus ou moins à l'actuel CNRS ». Pressés d'arriver à une décision, croyant avoir le pouvoir de décision sur le CNRS, les Sages pensaient déjà à établir la liste de la dizaine - tel est le chiffre donné par Pierre Aigrain, professeur à la faculté des sciences de Paris - de centres du CNRS qui répondaient à la définition «d'usines à recherche »), destinés à être rattachés à l'OCRST. Il va de soi que, même sur ce point, il était difficile d'aboutir à une liste définitive; d'où la proposition de cesser dans 
l'immédiat de discuter des nouveaux organismes et de réfléchir au statut des chercheurs, ce qui aurait permis, par la suite, de les affecter à l'un ou l'autre des organismes à créer. Dans la discussion qui suivit, les Sages se laissèrent emporter par une certaine surenchère dans les propositions. Sadron, par exemple, refusait fermement pour les chercheurs le statut de fonctionnaires; il proposait un statut de contractuels. On pensait déjà à la grille des rémunérations; elle aurait pu être calquée sur celle d'EDF et les chercheurs auraient cotisé à la caisse de retraite des cadres. Audelà des multiples solutions envisagées, tous étaient d'accord sur deux points: le nouveau statut devait offrir une carrière compétitive par rapport au secteur privé et les chercheurs devaient pouvoir être affectés aux laboratoires, selon les priorités de la recherche, et travailler aux côtés des universitaires, avec le statut de fonctionnaires. Même le principe d'un statut provoquait des dissensions au sein du CCRST sur des points comme le cumul ou la question de savoir s'il fallait faire de la place à une minorité de cadres fonctionnaires, le statut de fonctionnaire étant réservé à des cadres de haut niveau. Il fallait aussi décider des ministères de rattachement des nouveaux organismes. Sur ce point aussi il était difficile de faire l'unanimité. Il était sans doute plus facile d'apposer des veto que de faire l'unanimité sur une proposition de rattachement. D'où la décision suivante : «L'office des instituts nationaux de recherche (OINR) ne devrait être rattaché ni à l'Éducation nationale ni au délégué général mais en dehors de cela le point de rattachement importe peu. " Autre question: pouvait-on structurer la recherche appliquée sans pour autant réformer la recherche libre, sans réformer par conséquent l'Université ? Faute de pouvoir réformer rapidement les facultés des sciences, la création d'un office universitaire de la recherche scientifique (OURS) fut proposée ; il aurait eu le statut d'organisme de recherche et la possibilité de gérer le personnel CNRS affecté aux laboratoires universitaires.

Fin février 1959, si l'on résume les discussions au sujet du CNRS, la situation était la suivante : il y avait ceux qui pensaient plus en termes de gestion de chercheurs et ceux qui discutaient plutôt en termes d'organisation des organismes de recherche. Les premiers envisageaient de séparer les chercheurs en deux groupes : les uns enclins à s'engager dans une recherche de type universitaire, les autres plus attirés par les instituts de recherche appliquée. Les seconds pensaient en termes d'affectation des laboratoires aux nouveaux organismes de recherche et voulaient séparer le comité national de la gestion des laboratoires. Le comité national serait appelé à jouer le rôle d'expert auprès du Premier ministre. Comme le disait Germain, le nouveau CNRS serait alors composé de l'ensemble : fonds national-comité national rattaché soit au niveau du Premier ministre soit au Comité interministériel. Bref, on voulait transformer le CNRS en un organisme sans chercheur, un organisme de financement de la recherche.

Pendant ces discussions, les Sages n'avaient pas cru nécessaire de discuter avec le directeur du CNRS. Début février, ils lui demandaient de venir exposer ses idées sur le statut. Coulomb, craignant que l'octroi d'un statut ne soit pas le premier pas vers une réforme en profondeur de l'organisme, proposait que des mesures d'urgence soient prises pour l'ensemble du personnel. chercheurs, techniciens et administratifs. Malgré l'unanimité quant à l'urgence de la situation, et face à l'impossibilité de parvenir à une proposition unanime, Piganiol leur faisait savoir, indirectement, qu'il serait mieux de travailler à une solution qui pourrait entrer en vigueur le $1^{\text {er }}$ janvier 1960.

15 Entre-temps, peu d'attention avait été consacrée aux autres organismes. Maurice Letort du centre de recherche des charbonnages de France, président du CCRST, faisait état 
d'une demande du délégué soumise par une lettre du 16 février, invitant le CCRST à discuter de l'organisation de J'ensemble de la recherche, à partir d'un texte que Piganiol leur avait fait parvenir'. Prenant à contre-pied les projets réformateurs du CCRST, le délégué plaidait pour l'établissement d'interconnexions. À son avis, «la structure de la recherche en France se présentait en effet trop souvent comme une structure "à cellules fermées": on devait en faire une structure "à cellules ouvertes $»^{10}$. Ce texte proposait une réflexion générale sur la place et sur la diversité des recherches dont devait pouvoir disposer un pays comme la France. Si Piganiol semblait accepter certaines suggestions des Sages, comme la création de l'Office central de la recherche scientifique et technique, c'était pour mieux souligner le rôle indispensable de coordination plus que de centralisation des décisions qu'il fallait lui confier. Ce qui lui permettait, en conclusion de son document, de remarquer que probablement le CNRS avait connu des difficultés par rapport aux missions qui lui avaient été confiées à l'origine, dues à l'absence de statut convenable pour son personnel. Il ajoutait que si l'on voulait créer l'OCRST, tel qu'il avait été défini, il suffisait d'apporter quelques aménagements au statut du CNRS pour faire coïncider les deux organismes dans les faits. Fallait-il proposer aux ministres cette solution qui avait l'avantage de la simplicité ? Certes, remarquait Piganiol, le seul inconvénient de cette solution était que l'opinion publique n'aurait pas ressenti le même choc que celui provoqué par une décision changeant de fond en comble l'organisation de la recherche. Il s'empressait d'ajouter que " peut-être suffira-t-il d'une certaine propagande, qu'il y a lieu de définir ».

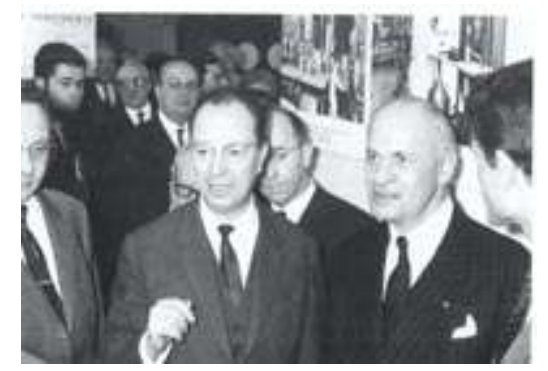

Pierre Jacquinot, directeur général du CNRS (1962-1969) en compagnie de Gaston Palewski, secrétaire d'État à la Recherche, le 15 décembre 1964 en visite à la $60^{\mathrm{e}}$ exposition de physique. Porte de Versailles à Paris. Photo CNRS.

Le premier comité interministériel de la recherche

Un texte sur les structures de la recherche fondamentale et le recrutement des chercheurs avait été rédigé par les services du délégué et soumis au CCRST pour accord. Ce texte résumait les diverses propositions avancées au cours des discussions multiples. Une organisation bicéphale était envisagée. Le statut "d'organismes de recherche " était proposé pour l'Office universitaire de recherche scientifique (OURS) et l'Office des instituts nationaux de recherche (OINR). Le rapport faisait état des discussions des statuts du CNRS, mettant encore une fois en évidence la nécessité d'assurer une carrière aux chercheurs. Les Sages avaient décidé de faire peu de cas des suggestions de Piganiol et de bien défendre leur projet qui reprenait dans ses grandes lignes celui de Germain.

17 En 1958, le personnel chercheur du CNRS était ainsi composé : 2328 chercheurs pour les sciences dites « exactes » et 663 pour les sciences humaines. La très grande majorité des chercheurs appartenait au grade d'aspirants-chercheurs (2 123 stagiaires et attachés de recherche), généralement des thésards; les chercheurs confirmés étaient 
868 et étaient régis par le statut de 1945, calqué sur celui de l'enseignement supérieur. Si le statut de chercheur ne semblait pas soulever de dissensions, celui des ingénieurs et des techniciens posait des questions sérieuses ${ }^{11}$. En 1957, sur 1135 postes d'ingénieurs ou de cadres recrutés au niveau de la licence, 376 n'en étaient pas pourvus et 167 étaient occupés par des agents n'ayant pas la qualification requise. Pire encore, 386 ingénieurs étaient partis pour le secteur privé pendant la seule année 1957, La conclusion était sans appel: l'insuffisance en nombre et en qualité du personnel technique des laboratoires était l'obstacle qui risquait de paralyser l'activité scientifique du Centre ${ }^{12}$. La direction du Centre avait réagi et avait proposé une refonte du décret du 28 mai $1952^{13}$. Le Comité consultatif avait refusé ce simple réaménagement du statut et penchait pour un statut complètement nouveau.

Les Sages pensaient avoir rempli leur mission et s'apprêtaient confiants à défendre leur projet devant le Comité interministériel de la recherche prévu pour le 23 avril. Entre temps, ils s'étaient occupés du Comité de recherches spatiales qui constituait le nouvel organisme à créer. Le jour même du Comité interministériel un pneumatique était adressé par les chercheurs de la Fédération de l'Éducation nationale (FEN) à Piganiol. Ils reprenaient une idée que l'on a trouvée dans son texte : le CNRS jouait déjà le rôle de l'OINR. Ils s'insurgeaient par conséquent contre le projet des Sages, qui essayait de substituer au "mécanisme bien rodé du CNRS qui arrivait à maturité, une nouvelle structure créée de toutes pièces $»^{14}$. Demandant un statut unique pour les chercheurs de tous les instituts de recherche, ils réclamaient un élargissement des attributions du CNRS, «lui permettant une plus grande efficacité dans la gestion des instituts de recherche».

Le premier Comité interministériel de la recherche scientifique et technique fut présidé par le Premier ministre, Michel Debré15. Une fois la séance ouverte Jacquinot prend la parole et définit ce que le gouvernement attend de la discussion: il faut «éviter de créer une administration nouvelle plus ou moins autoritaire et lourde», et il paraît préférable de se servir des « organismes existants dans la mesure où ceux-ci [ont] fait leurs preuves $»^{16}$.

Letort, en qualité de président des Sages, est alors invité à présenter les grandes lignes $\mathrm{du}$ projet du CCRST. À la demande du Premier ministre, Piganiol intervient sur le budget de la recherche et plaide pour une augmentation. L. Jacquinot et Pierre Guillaumat reconnaissent le bien-fondé de la demande en faveur du financement de la recherche. Guillaumat reproche alors au CCRST le caractère insuffisant de son travail car il s'est limité à J'analyse de la recherche fondamentale. Ce qui donne l'occasion à Boulloche d'intervenir pour critiquer le projet qui concerne le CNRS. Il ajoute que : « le CNRS [doit] conserver ses contacts avec l'industrie et les applications, sous peine de sclérose et d'absorption pure et simple par l'enseignement supérieur. Il fallait sans doute le modifier, l'améliorer, mais ne pas restreindre son action générale et, au contraire, la développer ». Il se déclare opposé à l'OINR et rappelle que réformer ne signifie pas pour autant une "condamnation à terme du CNRS ». Houdet et Chenot défendent l'autonomie des organismes placés sous la tutelle de leur ministère et se déclarent contre le démantèlement du CNRS, tout en souhaitant que des changements soient apportés à son organisation. 


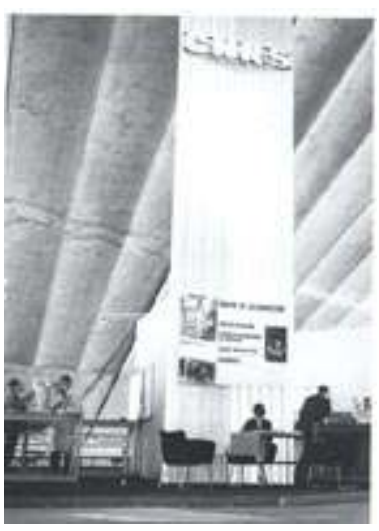

Vue d'ensemble du Centre de documentation du CNRS, 1954. Photo CNRS.

La discussion se déplace alors sur la création du fonds de la recherche et sur sa gestion. Il ne reste à Pierre Taranger, membre du CCRST, qu'à constater que ce comité interministériel sanctionne la défaite du CCRST car, aux propositions de changements, a été opposée "l'intangibilité des cadres existants ». On comprend bien l'amertume des Sages mais on peut aussi se demander si. à force de cogiter sur des réformes souhaitées, mais jamais réalisées, les membres du CCRST n'avaient pas perdu tout réalisme dans leurs propositions. Au manque de suivi des projets succédait la surenchère, concrétisée par des restructurations toujours plus radicales. De plus, en ayant fait du CNRS et de l'Université l'essentiel de leur réflexion, sans trop consulter le ministre concerné, ils ne pouvaient que susciter l'opposition de celui-ci, qui se voyait privé de fait de son pouvoir de décision.

Prenant la parole, le Premier ministre résume les décisions, avant de conclure. Il pense qu'un effort est possible pour satisfaire les demandes de crédits des chercheurs. « Pour le CNRS, il fallait le modifier et l'améliorer afin qu'il puisse notamment prêter son concours aux ministères autres que celui de l'Éducation nationale [...] Le bilan des recherches nationales devait être fait chaque année et le CNRS devait apporter un travail de synthèse. » Il demandait par conséquent à L. Jacquinot d'étudier les mesures à prendre en ce sens, propositions qui seraient soumises rapidement au Conseil des ministres. Somme toute, le CNRS sortait de cette première phase non seulement reconnu dans ses anciennes fonctions, mais renforcé car assuré dans son rôle d'organisme qui, seul, par le nombre de domaines touchés par ses laboratoires, pouvait avoir une vision du développement de la science et des priorités de la recherche. Certes, le fait qu'il était "évalué» par le CCRST le mettait à égalité avec les autres organismes de recherche, mais il gardait pleinement ses fonctions.

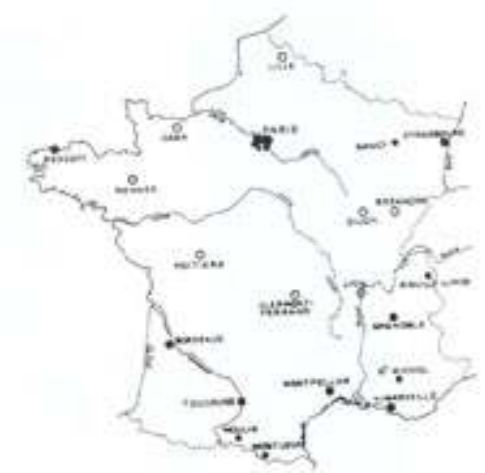

Carte de France avec emplacements des laboratoires du CNRS, 1954, Photothèque CNRS. 
En réalité, le sort de la réforme voulue par le CCRST avait été tranché lors d'une réunion informelle du 10 mars 1959, à laquelle avaient participé Étienne Bauer, chimiste, du cabinet du ministre de l'Éducation nationale, Piganiol et Raymond Poignant de la DGRST, et Pierre Lelong, conseiller scientifique à l'Élysée. En rendant compte des résultats de cette réunion, Bauer rapportait une critique de fond qui s'était manifestée vis-à-vis du projet du CCRST : «Accepter que le personnel chercheur soit mieux payé que le personnel de l'enseignement supérieur» paraissait être une solution au flux continu de jeunes docteurs quittant le CNRS pour l'industrie ou pour d'autres organismes publics de recherche. Mais le danger n'était pas exclu que s'instaure une sorte de surenchère entre chercheurs CNRS et enseignants-chercheurs de l'Université. Bref, il ne fallait pas créer des différences trop marquées entre le CNRS et l'Université, au moins au niveau du statut des chercheurs. Tout au plus pouvait-on imaginer l'attribution de primes dans certains cas, pour compenser la différence de salaire avec des centres de recherches analogues du secteur privé ou d'autres organismes publics. Dans une situation de pénurie de candidats, il était indispensable de sauvegarder l'équilibre entre organismes, sous peine de créer des situations difficiles à gérer.

Le statut des chercheurs

24 Le directeur du CNRS avait été convoqué par le CCRST le 29 avril 1959 pour savoir « dans quelles conditions le Comité national du CNRS pourrait éventuellement servir [...] à des fonctions de planification de la recherche en général ${ }^{17}$. Ce sujet n'ayant plus lieu d'être traité à la suite du Comité interministériel. L'audition de Coulomb se portait alors sur les modifications à apporter à la composition des commissions dans la perspective d'une réforme du CNRS. Coulomb fit savoir que, à la demande du ministre de l'Éducation nationale, une commission avait été instituée sur la question des rapports CNRS-enseignement supérieur. Il avait délégué deux représentants syndicaux : Raymond Dedonder, chercheur du CNRS, et Mlle Stora Coulomb se déclarait disponible pour collaborer à la rédaction du nouveau statut. Le directeur du CNRS jouait la collaboration avec son ministère de tutelle tout en se montrant disponible pour discuter de certains points avec le CC RST, maintenant que l'existence du Centre n'était plus la question à l'ordre du jour.

Les syndicats aussi intervenaient dans le débat sur l'avenir du CNRS. Ils avaient rédigé un mémorandum qui se plaçait dans la continuité de ce qui avait été décidé au colloque de Caen en 1956. Rappelant l'une des résolutions qui disait que, «sans une recherche fondamentale vivante et en pleine expansion, la recherche appliquée d'un pays, sans cesse surprise par "événement, devient vite tributaire de l'étranger, ils constataient le déséquilibre qui s'était établi entre le CNRS et les autres organismes de recherche. Leur analyse de la situation rejoignait, en partie, celle du CCRST : la médiocrité pesait sur la recherche. Ils demandaient par conséquent un effort pour la recherche fondamentale. Si le CNRS n'avait pas pu remplir la mission de coordination de la recherche fondamentale en France, la responsabilité en incombait aux autres ministères qui avaient fait preuve de peu de disponibilité pour collaborer. Ils revendiquaient pour le CNRS le rôle d'organisme ayant développé « la recherche fondamentale en France et, à ce titre, ne pouvant pas être séparé complètement et artificiellement de l'enseignement supérieur, Ils jugeaient que l'on ne pouvait pas dissocier expansion de la recherche et formation à la recherche. Voilà pourquoi ils étaient opposés à la participation des chercheurs aux premier et second cycles universitaires; en revanche leur participation était souhaitée au niveau du troisième cycle. Quant aux statuts, ils rappelaient 
l'engagement pris à Caen: assurer les mêmes avantages, la même indépendance, la même stabilité, les mêmes garanties morales qu'aux personnels de l'enseignement supérieur.

Sur la question du statut des chercheurs, le CCRST avait été invité à analyser les statuts de l'ensemble des organismes de recherche pour mieux évaluer leurs avantages et leurs insuffisances respectifs. Félix Trombe, membre du CCRST, avait été chargé du rapport préliminaire. Il en ressortait que la situation des chercheurs était mauvaise au CNRS comme à l'Institut national de l'hygiène (INH). En outre, la situation des techniciens était mauvaise partout. La discussion de ce rapport montre que l'on s'acheminait pour les chercheurs du CNRS vers une situation mixte prévoyant la fonctionnarisation pour certaines catégories et des contrats individuels pour la grande majorité. Il ne restait qu'à trouver une solution pour les techniciens.

La décision de principe concernant les nouveaux statuts du CNRS a été prise lors de la réunion interministérielle du 24 juin 1959, présidée par M. Debré18. Plusieurs textes portaient sur la question du statut des chercheurs, la composition des commissions et du directoire. Coulomb avait travaillé en étroite liaison avec son ministère de tutelle. Une proposition de Piganiol était acceptée : élargir le corps électoral des commissions pour diminuer ainsi la proportion élevée d'universitaires électeurs. La solution qui s'était dégagée reposait sur un équilibre délicat. D'une part, le CNRS ne devait pas s'inféoder à l'Université et rester confiné à une recherche de type uniquement universitaire. D'autre part, il ne devait pas non plus mettre en danger l'Université. C'était le prix d'une autonomie difficile qu'il fallait acquérir par la reconnaissance d'un rôle à jouer qui ne fût pas uniquement celui de soutien à l'Université ou à la recherche sur des objectifs immédiats. Cette solution était décidément différente de la position défendue par les Sages. Ils étaient restés prisonniers d'une vision dichotomique entre science fondamentale et science appliquée. Le choix qui s'était imposé était celui qui aurait permis au CNRS de s'affranchir de ce dilemme. Il ne restait au CNRS qu'à faire la preuve que la distinction couramment faite entre recherche fondamentale et recherche appliquée était obsolète. Autrement dit, il devait devenir un organisme de recherche du $\mathrm{XX}^{\mathrm{e}}$ siècle. C'est lentement, et non sans problèmes, que l'on reconnaîtra ce rôle particulier au CNRS. Par exemple, dans le deuxième numéro du Progrès scientifique, il était explicitement affirmé que "bien souvent d'ailleurs ses travaux [ceux du CNRS] concernent aussi bien la recherche pure que la recherche orientée en vue d'application dans des domaines scientifiques, extrêmement variés ${ }^{19}$ ». Il était nécessaire de rappeler ce rôle de temps à autre, car on risquait de l'oublier.

Une réforme et des statuts pour le personnel

Les 22 et 27 juillet 1959, le CCRST avait discuté les projets des nouveaux statuts pour les chercheurs du CNRS. Entre-temps, les douze avaient été occupés par l'élaboration des thèmes qui devaient constituer les sujets des premières actions concertées financées sur le fonds de développement de la recherche scientifique et technique. Lors du comité interministériel de la recherche du 28 juillet 1959, les projets de décrets concernant le CNRS étaient globalement acceptés. Le Premier ministre demandait qu'on lui fît parvenir dans le délai d'une semaine les dernières observations. Le 9 décembre 1959, un an près la mise en place des nouvelles structures chargées de coordonner la politique de la science en France, les décrets relatifs au fonctionnement du CNRS étaient publiés. Le CNRS était le premier organisme à bénéficier d'une 
réforme, ce qui, pour les chercheurs, signifiait enfin la possibilité d'une carrière en tant que chercheurs.

Les huit décrets et les quatre arrêtés de 1959 portent sur la composition du conseil d'administration et sur ses fonctions, sur l'organisation du comité national et sur la composition du corps électoral sur la structure et le fonctionnement du directoire. Quant au statut des chercheurs, quatre grades étaient créés : attaché de recherche, chargé de recherche, maître de recherche et directeur de recherche, Ces différents grades correspondaient à divers moments dans la vie du chercheur : le grade d'attaché était réservé à ceux qui devaient encore faire preuve d'aptitude à la recherche; pour accéder au grade de chargé, il fallait avoir obtenu un doctorat d'État ; étaient nommés maitres les chercheurs responsables d'une équipe ; le grade de directeur était réservé à ceux qui dirigeaient un laboratoire, Les attachés de recherche étaient engagés pour une période de deux ans, renouvelable deux fois, et une fois encore sur proposition du directoire, Les chercheurs appartenant aux autres grades étaient engagés pour une durée illimitée, Deux autres décrets instituaient deux corps. Le premier portait sur la création d'un corps de directeurs scientifiques dans lequel étaient admis les directeurs de recherche chargés de diriger une équipe et les directeurs et directeurs adjoints d'instituts de recherche. Le deuxième portait sur la création d'un corps de fonctionnaires participant à la gestion scientifique de laboratoires du Centre. La carrière des deux corps était calquée sur celle des professeurs universitaires et des maitres de conférences. En 1959, 440 chercheurs et 1525 techniciens étaient chargés du fonctionnement des laboratoires du CNRS, tandis que 2685 chercheurs et 1560 techniciens étaient mis à la disposition d'autres laboratoires, essentiellement universitaires.

Ces décrets marquaient la fin d'une phase dans la vie du CNRS, période pendant laquelle son existence avait été mise en cause par la vision généralement acceptée d'une séparation entre recherche fondamentale, donc universitaire, et recherche à partir d'objectifs. L'octroi au personnel d'un statut leur permettant de faire une carrière au CNRS enlevait l'une des justifications souvent invoquées pour justifier que le CNRS ne pouvait pas remplir ses missions.

Le rapport de conjoncture

31 Reconnu parmi les organismes de recherche comme ayant une mission particulière, le CNRS avait voulu et obtenu la mission d'effectuer, tous les ans, un rapport de conjoncture sur la recherche en France. En effet, dès le début de 1959, Coulomb avait invité les commissions du CNRS à réfléchir, sur le plan national. à préciser les besoins de chaque discipline scientifique et à envisager le développement souhaitable dans les années à venir, que les moyens nécessaires à ce développement soient fournis par le CNRS lui-même, l'enseignement supérieur ou par tout autre organisme ${ }^{20}$.

Présentant le premier rapport de 1959, Coulomb marquait la continuité avec la pratique, instituée depuis 1953, de réunir les sections du comité national en sessions extraordinaires pour "élaborer le programme des activités du CNRS, pendant les années suivantes $\rrbracket^{21}$. Ces discussions servaient de base aux travaux de la commission de la recherche scientifique et technique du Plan. Coulomb affirmait indirectement que le CNRS avait fait la preuve qu'il était en mesure d'accepter les défis que le pays posait en terme de recherche. Coulomb faisait remarquer que ce premier rapport de conjoncture reflétait effectivement l'opinion de la communauté scientifique, soit parce que les rapporteurs avaient mené une enquête auprès des collègues, indépendamment de 
l'organisme de rattachement, soit parce que la discussion avait été approfondie au sein des sections. Revendiquant pour le rapport de conjoncture un "panorama de la Science » avec très peu de lacunes, Coulomb reconnaissait l'impression d'hétérogénéité qu'en donnait sa lecture. Fallait-il chercher la cohérence à tout prix, ou laisser les fortes personnalités scientifiques exprimer leurs points de vue, même s'ils pouvaient apparaître contradictoires, quand on les lisait les uns à la suite des autres? Il disait préférer cette hétérogénéité à la cohérence obtenue artificiellement «lorsqu'on s'adresse à des secrétaires. »

L'objectif au départ était double : permettre aux sections de distribuer les crédits en connaissance de cause, refusant l'équipartition comme critère de facilité ; planifier le nombre de postes de chercheurs recrutés dans les diverses disciplines. Un objectif à plus long terme était celui d'ouvrir un dialogue avec les scientifiques sur les perspectives de leurs disciplines. Coulomb reconnaissait qu'il était difficile de synthétiser un document qui accentuait l'aspect mono disciplinaire, selon les sections du comité national. Mais il s'agissait d'un premier exercice, sans doute à améliorer.

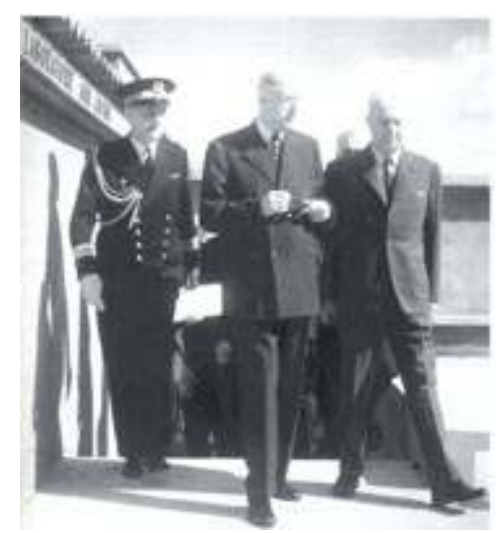

Le général de Gaulle en visite au laboratoire Aimé-Cotton, le 18 mars 1965. CNRS-D. Fautret.

Une analyse des différents rapports de conjoncture donnerait non seulement l'état de la situation des diverses disciplines, mais aussi les souhaits des chercheurs en divers domaines et les listes des instruments qu'ils pensaient être nécessaires pour mener une recherche dynamique. Il s'agissait aussi de l'apprentissage d'une pratique que les scientifiques n'avaient pas l'habitude d'utiliser sous cette forme. D'ailleurs, on trouve des remarques, comme celles sur la nécessaire garantie de la liberté du chercheur et de l'indépendance dans le choix des sujets de recherche, qui montrent la difficulté à trouver une méthode de travail dans le cadre de la prospective. Mais le problème de la coordination avec les champs de recherche proches se posait déjà, car les divisions constituaient une sorte de contrainte artificielle; d'où la suggestion de créer des inter commissions. Il est aussi intéressant de noter que l'on pensait, à Paris, pouvoir influencer la province, en nommant des directeurs et des maîtres de recherche, à la condition qu'ils quittent la capitale, La décentralisation et la promotion associées étroitement ne sont pas une invention récente. Il reste que l'on possède dans ces rapports un inventaire de l'état de la recherche en France, très détaillé. Si le premier rapport constituait la nouveauté, les autres avaient déjà un texte qui malgré ses défauts leur servait de comparaison pour évaluer ce qui avait eu une suite dans les réalisations et ce qui était resté à l'état de projet.

Les liens CNRS/Université reviennent à l'ordre du jour 
fois le Centre reconnu comme appartenant aux organismes de recherche dotés d'une mission particulière, la question de ses rapports avec l'Université resurgissait en particulier lors de l'examen de l'enveloppe consacrée à la recherche par les Sages. Depuis la création du fonds pour la recherche, trois sources de financement permettaient de développer la recherche: la première constituait le mode de financement typique de l'Université et était destinée essentiellement aux chaires, à des hommes donc. La deuxième prenait comme critère le développement par discipline et était la forme suivie par le CNRS. La troisième, celle du fonds, faisait l'objet d'une répartition par problème posée ${ }^{22}$. La question était de savoir s'il y avait recouvrement entre ces trois sources de financement et s'il fallait l'accepter. Devait-on augmenter le mode de financement par contrat, laissant seulement à l'Université le principe d'un financement des hommes? Mais l'Université aussi avait des instituts de recherche. De plus, l'augmentation des crédits universitaires diminuait l'importance du financement individuel apporté par le CNRS. Bref, encore une fois la question des frontières se posait, mais sous la forme de la responsabilité d'une politique qui revenait au ministère et à ses directions.

Pour faciliter le débat, un rapport avait été confié à Bernard Vodar, directeur du laboratoire CNRS des ultra hautes pressions. L'essentiel du rapport portait sur la nécessité d'établir une coordination entre la direction de l'enseignement supérieur et la direction du CNRS, alors que les deux directions fonctionnaient plutôt en concurrence. Or l'une des fonctions du CCRST était d'harmoniser les crédits alloués à la recherche. Plusieurs indices montraient que les laboratoires échappaient à cette coordination, car les laboratoires, surtout universitaires, avaient la possibilité de jouer «sur plusieurs tableaux ». La seule manière d'éviter cela, selon Vodar, était de créer des instituts de recherche destinés à faciliter une recherche systématique fondamentale des « domaines encore vierges $»^{23}$. André Giraud, pour sa part, constatait que les mêmes créations de laboratoires étaient proposées par l'enseignement supérieur et par le CNRS. Cette situation était inconcevable pour les membres du CCRST. Comme à l'habitude, il y avait ceux qui prônaient un rapprochement étroit, suggérant par exemple que CNRS et enseignement supérieur dépendent d'un même directeur au ministère de l'Éducation nationale. D'autres préféraient une séparation nette avec l'attribution de compétences bien définies. Ils énonçaient même le principe que «l'enseignement supérieur est fait pour enseigner et le CNRS pour faire de la recherche $»^{24}$. Il découlait de ce principe que les crédits auraient dû être affectés à l'Université en fonction des besoins d'enseignement et au CNRS en fonction des nécessités de la recherche.

On discutait sans fin de ces questions au sein du CCRST. Certains voyaient dans la politique menée par le CNRS une limitation de la liberté totale dont jouissaient les universitaires dans le choix de leur champ de recherche. Parfois, les distinctions entre CNRS et Université étaient simplistes. La structure essentielle de l'Université demeurait la chaire, alors que le laboratoire constituait l'élément fondamental du CNRS. Plus dynamique, le CNRS pouvait être destiné à corriger «les défauts naturels et inéluctables de l'enseignement supérieur, particulièrement en ce qui concernait la création d'enseignements nouveaux qu'il devait, après une période, passer à l'Université $»^{25}$. Au fur et à mesure de ces discussions, certaines insuffisances dans le statut du CNRS, en particulier en ce qui concernait la constitution des commissions du comité national. commençaient à apparaître. 

Dans le cas de celui portant sur le CNRS et l'Université, il choisissait de suggérer l'interchangeabilité des personnels en confiant aux chercheurs aussi des tâches d'enseignement et proposait pour les professeurs l'année sabbatique ${ }^{26}$. Un consensus au moins sur deux points était à l'origine de ce vœu :

b) il fallait distinguer entre la recherche relevant du libre choix du professeur et la nécessité de laboratoires destinés à exécuter des recherches systématiques. À la lecture des comptes rendus de ces discussions, on découvre que les problèmes étaient toujours là et resurgissaient périodiquement. Il est aussi intéressant de constater que l'on raisonnait toujours selon l'idée d'une planification maximale, c'est-à-dire que l'on craignait que deux laboratoires pussent travailler, en compétition, sur des domaines proches. Signe aussi que la réforme de 1959 n'avait pas encore été acceptée, faute d'une réforme universitaire. Il était toujours facile d'opérer des distinctions de principe. Concrètement, il devenait problématique de savoir quand on pouvait parler de financement régulier et quand on devait envisager un financement exceptionnel. L'opinion de Coulomb était la suivante: attribuer «des crédits réguliers aux professeurs d'enseignement supérieur en fonction du nombre de chercheurs » dirigés ou rattachés à la chaire ${ }^{27}$. Certes, cette solution était rendue plus facile du fait que l'on était en période d'expansion du financement de la recherche. On commençait à parler des universités comme des institutions ayant une vocation régionale, invitées donc à se servir de tous les ressorts offerts par les situations locales, alors que le CNRS gardait plutôt une ambition nationale.

Les laboratoires associés

41 Encore une fois, les questions statutaires provoquèrent le changement. Un problème se posait avec récurrence : les chercheurs rattachés à une chaire se trouvaient parfois en difficulté quand la direction des équipes universitaires changeait. Il fallait trouver une solution. Certes, le poids de l'histoire était important pour cette question. En 1962, on comptait 2566 chercheurs du CNRS mis à la disposition des facultés ou des laboratoires de l'enseignement supérieur (droit 53, lettres 364, sciences 1599, médecine 250, pharmacie 150).

42

Début juillet 1962, J. de Chalendar, inspecteur des Finances, présentait devant le CCRST l'état d'avancement des travaux de la mission recherche qui lui avait été confiée dans le cadre du ministère de l'Éducation nationale. C'était le début d'une série d'auditions. Il commençait par rappeler certains problèmes techniques, comme les questions liées à la TVA ou à la possibilité pour les entreprises d'inclure les contrats de recherche parmi les frais généraux, ou encore la déduction possible des versements effectués au profit de sociétés ou d'organismes publics dans la limite de 1 pour mille du chiffre d'affaires. Il critiquait de manière courtoise le fonctionnement des Sages, en leur suggérant de communiquer les vœux émis en priorité aux organismes intéressés, en leur demandant de réagir dans un délai raisonnable. La question de l'assouplissement des normes budgétaires et de la décentralisation de l'administration était posée, sans pour autant recevoir une réponse définitive de la part de De Chalendar. 


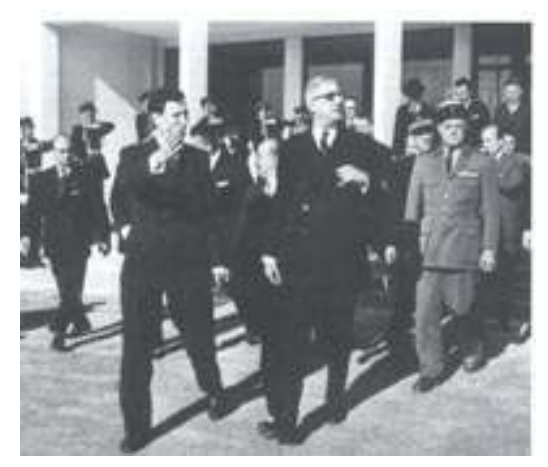

Le général de Gaulle et Jean Coulomb, directeur général du CNRS. CNRS-Ray Delvert.

Lors de la deuxième audition, le 20 juillet 1962, il était question essentiellement du CNRS. La mission conduite par de Chalendar faisait remarquer dans ses conclusions que le CNRS, surtout en sciences humaines, suivait peu les priorités fixées au niveau national. Le IV ${ }^{e}$ Plan, par exemple, prévoyait le développement de la sociologie et de la démographie, ce qui n'avait pas été le cas au sein du CNRS. Cette remarque soulevait la question de savoir si le CNRS devait mener de manière autonome sa politique de recherche ou s'il devait mener sa politique d'expansion dans le cadre des priorités nationales. Ce suivi devait-il se faire par la mise au concours de postes «fléchés » dans les disciplines prioritaires, ou fallait-il le faire en restructurant des laboratoires. Ce qui conduisait à la question de l'évaluation régulière des laboratoires et du redéploiement éventuel du potentiel de chercheurs et de techniciens. Fallait-il reconvertir des laboratoires effectuant des recherches dont l'intérêt aurait diminué avec le temps, pour concentrer les moyens sur : a) « les disciplines d'avant-garde, en créant, au besoin, des laboratoires pour des périodes de temps limitées, et en les remettant ensuite aux facultés, dès que les disciplines correspondantes feront l'objet d'un enseignement universitaire régulier $" ; b)$ des installations très importantes et onéreuses dépassant les moyens d'une faculté, pouvant être utilisées par plusieurs facultés d'une même région, en accord avec les priorités fixées par le Plan, par le CCRST ou par la délégation. C'est une vision d'un CNRS à la fois répondant aux priorités de la politique nationale et gestionnaire d'équipements lourds de recherche. Il y avait une certaine contradiction entre les deux objectifs. Comment modifier les objectifs de recherche des laboratoires universitaires auxquels étaient affectés la majorité des chercheurs CNRS ? Quant aux relations CNRS/Université, le rapport de De Chalendar suggérait de les garder distincts l'un de l'autre.

Le rapport de De Chalendar coïncidait avec le changement de direction du CNRS. Coulomb quittait le CNRS et était remplacé par Pierre Jacquinot. Le nouveau directeur était invité par les Sages à venir exposer la situation du recrutement des chercheurs. L'argumentaire de P Jacquinot reposait sur un inventaire exhaustif de l'affectation des chercheurs. On découvrait ainsi que des chercheurs CNRS travaillaient non seulement au sein des universités mais aussi auprès d'autres organismes de recherche. C'est à l'occasion d'une enquête sur les sciences humaines, que l'on avait découvert les liens entretenus avec d'autres organismes, comme l'ORSTOM. Pour renforcer ces liens, devait-on conseiller au CNRS de développer certaines disciplines, comme le groupe ASDE (Anthropologie, Sociologie, Démographie et Économie), peu représentées au sein de l'Université ? Cet événement était important car il relativisait la question des liens Université/CNRS et les plaçait dans le cadre plus général des liens avec les autres lieux de recherche. N'était-ce pas le rôle du CNRS que d'apporter son soutien aux projets de 
recherche novateurs ? Ce qui permettait de reprendre, sous un angle bien différent, la question de la place du CNRS dans l'ensemble de la recherche en France.

Le climat avait changé. On était passé d'un rapport tendu, qui caractérisait les débuts du CCRST. On était passé d'un rapport tendu, qui caractérisait les débuts du CCRST, à une sorte de collaboration. C'est ainsi qu'après avoir donné une sorte de satisfecit à l'action menée par le CNRS depuis son origine, après avoir reconnu sa capacité d'autoadaptation, en particulier avec la mise en place des recherches coordonnées sur programme (RCP), sur le modèle des actions concertées, les Sages reconnaissaient que le Centre avait des difficultés à faire de la prospective afin de répondre aux besoins interministériels. Il lui était donc difficile d'exercer un réel poids d'orientation de la recherche en France.

Ne pourrait-on considérer que le CNRS aurait dans l'avenir une mission d'orientation dans le cadre du ministère de l'Éducation nationale? Il faudrait pouvoir cibler, en matière d'objectifs de recherche fondamentale, les disciplines à soutenir en priorité. En ce sens, la notion d'orientation devait être comprise comme un moyen permettant de faire "connaître les disciplines ou les secteurs scientifiques qui doivent être développés $»^{28}$. En contrepartie, la direction de l'enseignement supérieur reconnaissait qu'un effort de clarté devait être fait pour connaître les divers canaux de financement dont pouvaient disposer les laboratoires universitaires. Autre sujet récurrent : la liberté du chercheur. La liberté du chercheur et la liberté de la recherche devaient être gardées intactes. Ce qui impliquait aussi une certaine planification pour l'enseignement supérieur. On abandonnait définitivement la vision du chercheur comme d'un homme qui travaille seul à la poursuite de ses hypothèses. Désormais la recherche était structurée en équipes, parfois autour d'appareils lourds et coûteux.

P. Jacquinot misait sur une croissance de l'emploi et sur un accroissement sensible des promotions à l'intérieur de l'organisme, en demandant la création de 800 postes par an pour les chercheurs et de 1900 postes de techniciens. Autre objectif poursuivi : fidéliser les chercheurs qui avaient tendance à partir souvent pour l'Université. Il considérait que le CNRS devait remplir la double mission «de recherche et de formation des chercheurs ", surtout qu'il devait donner des moyens d'expansion aux équipes valables et non pas viser seulement le maintien en activité d'équipes modestes ne pouvant atteindre que difficilement le niveau international ${ }^{29}$. La stratégie de P. Jacquinot est claire ; il demandait des moyens et en contrepartie, il proposait de mettre en place une évaluation sur la qualité de la recherche, au moins au sein de son organisme. Il considérait ces deux problèmes comme étroitement liés. C'est pourquoi il annonçait une série de mesures qui allaient dans le sens d'une évaluation des équipes. D'où la précision qu'il apportait: "Ayant constaté certaines insuffisances de liaison entre les laboratoires propres du CNRS et les commissions, il est envisagé d'associer à tous les comités de direction de ces laboratoires des représentants de commissions. Par ailleurs, sur un plan général, une "reconstruction» complète des comités de direction des laboratoires propres est prévue afin d'en stimuler l'activité.» On s'acheminait donc vers une évaluation généralisée des laboratoires propres. P. Jacquinot considérait nécessaire d'organiser un suivi constant des laboratoires. D'où l'idée de créer un système de parrainage pour mieux informer les membres des commissions et des «fiches de laboratoires " permettant à tous les membres des commissions d'avoir une vue synthétique des laboratoires évalués. Si l'évaluation devait être généralisée, il fallait régler la situation de ces chaires auxquelles avaient été affectés des chercheurs 
et des techniciens CNRS. C'est pourquoi il annonçait l'étude d'une «formule de laboratoires associés qui devrait se révéler plus efficace encore que le système des fiches de laboratoires. En effet, tous les laboratoires, en partie subventionnés par le CNRS, seraient liés au Centre par une convention régulièrement reconduite précisant... les commissions, la disponibilité, et les allocations contractuelles perçues par ces laboratoires ». Bref, j'idée d'équipes associées était en cours d'élaboration, le CNRS prenant l'initiative d'évaluer les chercheurs qui lui étaient rattachés. Les discussions interminables au sein du CCRST n'avaient pas permis de dégager une idée qui se révélait naturelle une fois abordé le problème de l'évaluation. Il y avait là, la clé de la nécessaire coordination entre université et CNRS.

Le CNRS : un organisme adapté aux nouveaux défis de la recherche

C'était le moment aussi de faire des bilans. Il paraissait globalement positif à Poignant qui remarquait l'importance prise par le rapport de conjoncture du CNRS, la mise en place de critères d'évaluation et l'harmonisation des budgets de la recherche. Quant aux liens CNRS/Université, après les laboratoires associés, il fallait probablement prévoir aussi des instituts mixtes. Face au développement des chaires et des maîtrises de conférence pour suivre l'évolution démographique, le danger était la multiplication de petites équipes associées aux chaires. Or, le CNRS par le biais de l'association permettait une évaluation dont l'enseignement supérieur était incapable, faute de structures adéquates ${ }^{30}$.

49 L'introduction de l'évaluation conduisait aussi à la définition de priorités dans l'embauche des jeunes chercheurs. Ainsi, pour l'année 1965, la croissance devait être prioritaire pour les mathématiques appliquées, pour la géophysique, pour certains secteurs de la chimie organique, la chimie biologique, la biologie cellulaire et dans certains domaines de la biologie animale. Certains secteurs devaient être mieux pourvus, comme l'électrotechnique. Il est intéressant de remarquer qu'on retrouve des priorités qui sont affichées aussi dans le cadre des actions concertées financées par le fonds de développement. Autre conséquence de l'évaluation : il était décidé de fixer à 8 ans la limite pour siéger sans interruption au comité national.

Une lettre de Valéry Giscard d'Estaing, sous-secrétaire d'État aux Finances, mettait fin à une phase d'expansion de la recherche: il faisait savoir qu'aucune nouvelle mesure n'était prévue, sur le plan du budget, dans la loi de finances pour 1965. Maintenant il s'agissait de défendre la recherche en son ensemble. Ce qui changeait radicalement le contexte des discussions, car on passait de la phase d'expansion à une nouvelle phase de restriction. L'évaluation et la reconversion des équipes devenaient des actions encore plus importantes en période de diminution des crédits et des postes de chercheurs et de techniciens.

51 Le climat a changé avec le coup d'arrêt dans la croissance des emplois et des crédits, intervenu en 1965. Désormais, il s'agissait avant tout de consolider ce qui avait été acquis et d'essayer d'assurer une certaine croissance. Devait-on considérer que la recherche n'était plus une priorité comme le montrait la nomination d'un secrétaire d'État à la Recherche à la place d'un ministre ? Au moment où le Ve plan prévoyait un important investissement dans les structures des laboratoires, par la construction de nouveaux bâtiments et .l'équipement de laboratoires anciens, la coordination semblait tout aussi importante. D'où la décision de créer au sein du ministère de l'Éducation nationale le Comité de coordination de la recherche scientifique. Il était présidé par le ministre de l'Éducation nationale et le secrétaire général de l'Éducation nationale en 
était le vice-président; les membres étaient le directeur général et le directeur administratif et financier du CNRS, le directeur des enseignements supérieurs et le délégué général à la recherche scientifique et technique, pouvant se faire assister par leurs collaborateurs. Un secrétariat permanent permettait de donner une continuité aux activités de coordination du nouveau comitée ${ }^{31}$.

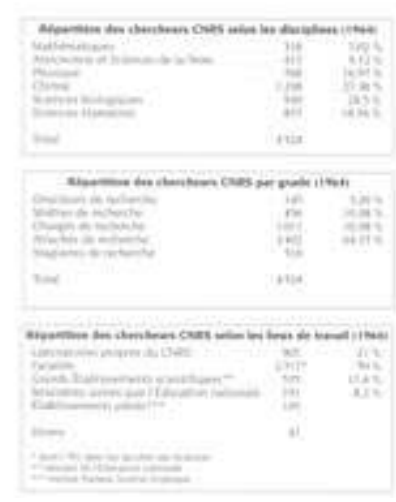

Le progrès scientifique, $\mathrm{n}^{\circ}$ 67, 1964.

Le CNRS était renforcé dans son autonomie administrative par un ensemble de décisions (décret du 31 mars 1966) qui complétaient son organisation, étant donné l'expansion qu'il avait connu et allait connaître. Le directeur général était épaulé par un directeur administratif et financier et par des directeurs scientifiques. Ensemble, ils constituaient le comité de direction. Ayant constaté le succès des RCP, à l'image de la DGRST, il était prévu la création de commissions interdisciplinaires, composées de membres choisis parmi ceux des commissions disciplinaires et de personnalités nommées ${ }^{32}$. Il était en outre prévu la possibilité de créer au sein du CNRS des instituts nationaux de recherche, Le critère de leur création était essentiellement la nécessité de disposer d'un équipement lourd. Leur création aurait été faite en regroupant plusieurs laboratoires déjà existants. La création de laboratoires de recherche associés était réglementée. Soumis à J'évaluation des commissions du comité national. il s'agissait d'un contrat de quatre ans engageant le CNRS et l'organisme de rattachement principal du laboratoire pour le développement de recherches innovantes. Une période prenait fin. Elle avait donné une physionomie nouvelle au CNRS. Désormais, sa structure semblait correspondre aux missions qui lui avaient été assignées. Or, si l'on regarde ce qui avait été fait jusque-là, il s'était agi de mettre de l'ordre dans une situation un peu confuse où le CNRS et l'Université étaient très liés. C'est pourquoi, au terme de sept ans de réformes, le CNRS est devenu un soutien important de l'Université tout en obtenant pour son personnel un statut qui lui permettait d'envisager une carrière. 


\section{NOTES}

1. Cette appellation était couramment attribuée aux membres du CCRST pour signaler qu'ils siégeaient à titre personnel et non en tant que représentants de leurs institutions d'origine dont ils auraient da défendre les intérêts.

2. C. Nicolet, «La science dans la République », Cahiers de la République, janvier-février 1957.

3. AN-81/141. La lettre de mission définissait ainsi les objectifs : «Établir un rapport sur le fonctionnement administratif de la recherche scientifique, en vue de rechercher les modifications et les assouplissements qui peuvent être apportés aux règles actuelles de gestion des crédits, notamment en ce qui concerne le fonctionnement des laboratoires. »

4. L'une des conséquences de cette situation incertaine était que les techniciens quittaient le Centre pour entrer dans le secteur privé et que les jeunes thésards choisissaient ce secteur plutôt que le CNRS. Si la mobilité était jugée positive car elle permettait de transférer des chercheurs compétents vers les entreprises, en revanche les laboratoires étaient pénalisés par ces départs, de deux manières. Lune était la perte de personnel formé, au moment où celui-ci devenait opérationnel, l'autre était le faible recrutement de personnes qualifiées. Le rapporteur pour en signifier l'importance reprenait ces points dans les conclusions pour mieux souligner le danger d'une telle situation.

5. Ch. Sadron. Recherche scientifique. Université et CNRS. document du 9 décembre 1958. distribué aux membres du CCRST. AN- 141.

6. A. Lichnérowicz, Remarques sur les structures de la recherche fondamentale. AN-81/141.

7. Lettre de Charles Sadron à Maurice Letort, président du CCRST, du 7 février 1959, AN-81/141.

8. Compte rendu de la réunion du CCRST du 25 février 1959, AN 81/121.

9. Mise au point récapitulative de M. Letort, du 18 février 1959, AN-81/141.

10. P. Piganiol. La recherche scientifique dans la nation, texte du 16 février 1958, AN-81/141.

11. Pour ces chercheurs, deux statuts étaient envisagés : celui de contractuel selon la grille d'EDF ou du CEA, ou celui de fonctionnaire, selon la carrière dans l'enseignement supérieur. Les titulaires auraient été affectés au corps des CNRSU (Centre national de la recherche scientifique universitaire), les autres auraient rejoint les OINR. Il y aurait donc eu séparation des chercheurs en deux corps, ce qui aurait sans doute vidé le CNRS. 12. R. Poignant, conseiller technique auprès du délégué général. Rapport sur les structures de la recherche fondamentale et le recrutement des chercheurs, AN-81/141. 13. La direction proposait une augmentation des indices terminaux, un assouplissement des règles de recrutement, une gestion des carrières personnalisée. La rémunération serait fixée par le directeur dans une fourchette définie. Des indemnités spéciales étaient prévues.

14. Pneumatique adressé à M. Piganiol le 23 avril 1959 par A. Kepes, secrétaire adjoint du Bureau du Syndicat national des chercheurs scientifiques.

15. Assistaient à la séance outre les membres du CCRST et Piganiol. MM L. Jacquinot, ministre d'État, Guillaumat, ministre des Armées, Boulloche, ministre de l'Éducation 
nationale, Houdet, ministre de l'Agriculture, Chenot, ministre de la Santé publique et de la Population, Giscard d'Estaing, secrétaire d'État auprès du Premier ministre et le représentant de l.-M. Jeanneney, ministre de l'Industrie et du Commerce.

16. Procès-verbal de la réunion du Comité interministériel de la recherche scientifique et technique du 23 avril 1959, AN-81-121.

17. AN-81/12. CCRST-Compte rendu de la réunion du 29 avril 1959.

18. Participaient à cette réunion outre le Premier ministre, Jacquinot. Boulloche, Jeanneney, Chenot, Giscard d'Estaing, le général Lavaud, représentant le ministre des Armées, Ferru, directeur de l'INRA, représentant le ministre de l'Agriculture, Piganiol et les membres du CCRST à l'exception de Lichnérowicz.

19. «Schéma de l'organisation de la recherche scientifique et technique française », Le progrès scientifique, 2, avril 1961.

20. Cité dans « L'évolution statutaire du Centre national de la recherche scientifique (CNRS) », Le progrès scientifique, 5, mai 1961.

21. CNRS, Rapport de conjoncture, Introduction, 1959.

22. CCRST, réunion du 14 octobre 1960.

23. B. Vodar, Les relations CNRS/Université. Tentative d'analyse de la situation actuelle. AN-81/158. Plusieurs critères justifiaient l'avantage des instituts : les connexions interdisciplinaires facilitées ; les moyens peuvent être importants ; à partir d'une recherche très abstraite (fondamentale) les ramifications jusqu'aux applications pourraient être organisées au sein du même établissement ; les moyens en personnels pourraient être importants. Ces instituts pourraient héberger des laboratoires techniques et des laboratoires d'objectifs.

24. A. Giraud, Note sur les relations CNRS/Université. AN-81/158.

25. AN - 81/123. Compte rendu de la réunion du 10 février 1961.

26. CC RST, Projet de vœu du CCRST sur le problème de relations CNRS/enseignement supérieur, 28 avril 1961.

27. Audition de Coulomb par le CCRST. Séance du 14 avril 1961. AN-81/123.

28. Compte rendu de la réunion du 21 février 1964. AN-77/321-323/1780.

29. CCRST. Réunion du 10 avril 1964. AN-81/126.

30. R. Poignant. Structures françaises de recherche, AN-81/154.

31. Le premier secrétaire a été Jean-Claude Siebauer, auditeur à la Cour des comptes.

32. En 1964, il existait 55 RCP dotées d'un budget de 4,7 millions de francs.

\section{RÉSUMÉS}

CNRS: Higher Stake in France's Scientific Research Policy

On December 9, 1959, eight government decrees and two ministerial orders were published in the Journal Officiel. They were ail about the CNRS and resulted from one year of debates within the Consultative Committee of Technical and Scientific Research; the General Delegation for Technical and Scientific Research, and within the ministeries supervising the CNRS. G. Ramunni's analysis brings to light the range of various points of view aired within the debates, and the common ambition to turn the CNRS into a research organization with an independent research 
policy. He describes how these discussions took on a rather acrimonious tone when General de Gaulle came to power in May 1958. The decrees regulated the researchers' careers and made the CNRS in charge of drawing up an overall report on the state of research. They therefore established the CNRS as the central element of research organization in France.

\section{AUTEUR}

\section{GIROLAMO RAMUNNI}

Girolamo Ramunni est professeur d'histoire des sciences à l'université de Lyon II. Il a publié Les sciences pour l'ingénieur : histoire du rendez-vous des sciences et de la société, Paris, CNRS ÉDITIONS, 1995. 